\title{
Variability of Faecal Calprotectin in Inflammatory Bowel Disease Patients: An Observational Case-control Study
}

\author{
Anneline Cremer, ${ }^{\mathrm{a}, \mathrm{b} *}$ Jade $\mathrm{Ku}{ }^{a *}$ Leila Amininejad, ${ }^{\mathrm{b}}$ \\ Marie-Rose Bouvry, ${ }^{\mathrm{C}}$ Fabian Brohet, ${ }^{\mathrm{c}}$ Claire Liefferinckx, ${ }^{\mathrm{a}}$ \\ Jacques Devière, ${ }^{\mathrm{a}, \mathrm{b}}$ André van Gossum, ${ }^{\mathrm{b}}$ Julie Smet, ${ }^{\mathrm{c}}$ Patrick Stordeur, ${ }^{\mathrm{c}}$ \\ Denis Franchimont ${ }^{\mathrm{a}, \mathrm{b}}$
}

aLaboratory of Experimental Gastroenterology, Université Libre de Bruxelles, Brussels, Belgium ${ }^{\mathrm{b} D e p a r t m e n t}$ of Gastroenterology, Erasme University Hospital, Université Libre de Bruxelles, Brussels, Belgium 'Immunobiology Clinic, Erasme University Hospital, Université Libre de Bruxelles, Brussels, Belgium

*Sharing first authorship.

Corresponding author: Anneline Cremer, MD, Department of Gastroenterology, Erasme University Hospital, Route de Lennik, 8081070 Bruxelles, Belgium. Tel.: +32 2555 5773; Fax: +32 2 5554697; Email: anneline.cremer@erasme.ulb.ac.be

\begin{abstract}
Background and Aims: Several factors have been reported to affect faecal calprotectin $[F C]$ values, and significant variation in FC concentrations has been observed in inflammatory bowel disease [IBD] patients. We aimed to evaluate FC variability in IBD patients, and to assess the robustness of a single stool punch.

Methods: This is a single-centre observational case-control study. Disease activity was assessed using endoscopic and clinical activity scores, as well as C-reactive protein levels. Stool samples were collected twice within a 1 to 6 days interval, and FC was measured on punches and homogenates by fluorometric enzyme immunocapture assay.

Results: In all, 260 stool samples were collected from 120 patients. Intrastool variability was low, with an intraclass correlation coefficient for single measures between three punches from a single stool sample of 0.91 , and median coefficient of variation [CV] of $17 \%$. CV of two stool samples a few days apart [intra-individual variability] were significantly higher [ $p<0.01]$ with median CV of $36 \%$. FC standard deviations correlated with mean FC levels either for intrastool or for intra-individual variability, with a Spearman's coefficient of rank correlation of 0.85 and 0.78 , respectively $[p<0.01]$. Disease type, location, activity, and FC levels did not influence variability.

Conclusions: A single stool punch is reliable for FC measurement, considering that intrastool variability is low. Intra-individual variability a few days apart is significantly higher. Therefore, decision-making strategies based on single measurements should consider this variability, to determine the minimum optimal variation to be achieved, rather than a cut-off, especially in high FC levels.
\end{abstract}

Key Words: Faecal calprotectin; variability; inflammatory bowel disease 


\section{Introduction}

The heterodimeric calcium- and zinc-binding protein, calprotectin, mainly found intracellularly in neutrophils, accounts for about $60 \%$ of cytosolic proteins of these cells. ${ }^{1}$ Its level in faeces is proportional to the amount of neutrophil migration into the bowel lumen from the inflamed wall., ${ }^{2,3}$ Faecal calprotectin $[\mathrm{FC}]$ is therefore a reliable biomarker of intestinal inflammation, ${ }^{3-8}$ and is currently the most sensitive noninvasive surrogate marker of disease activity in inflammatory bowel disease [IBD]. FC is nowadays widely used in the management of IBD patients. ${ }^{9}$

The differential diagnosis of IBD from irritable bowel syndrome [IBS] may be difficult based on clinical symptoms only, due to nonspecific clinical presentation of IBD, especially in Crohn's disease $[\mathrm{CD}] . \mathrm{FC}$ is a valuable non-invasive diagnostic and monitoring marker in IBD patients, limiting the number of unnecessary endoscopies. Although there is no strong correlation between clinical scoring systems for IBD and FC levels, FC has a strong correlation with endoscopic and histological remission both in $\mathrm{CD}$ and in ulcerative colitis $[\mathrm{UC}] .{ }^{10} \mathrm{FC}$ is increasingly used for patients in clinical remission to predict disease relapse ${ }^{11-13}$ or mucosal healing, and in active disease to monitor response to therapy. ${ }^{14-16}$

FC values depend on disease type [CD or UC], disease activity [quiescent or active disease], and disease location. FC is more sensitive in assessing disease activity in UC than in $\mathrm{CD},{ }^{17}$ whereas in $\mathrm{CD}$ it is more sensitive for colonic or ileocolonic involvement compared with isolated ileal involvement. ${ }^{14}$ However, considerable overlaps in FC values exist among patients with different disease severity profile $^{14,18}$ and several studies found no correlation between baseline FC and response to treatment. ${ }^{15,19,20}$

FC is highly resistant to bacterial and enzymatic degradation and is considered to be homogeneously distributed throughout the stool. ${ }^{21}$ In the presence of calcium, FC is stable in faeces at room temperature for up to 7 days. ${ }^{2,3,22}$ However, several factors have been reported to affect FC values, and a significant day-to-day ${ }^{23,24}$ and within-day ${ }^{25}$ variation in FC concentrations has been observed in IBD patients, mainly in subgroups of patients [UC or CD and quiescent or active disease]. Known factors are colonic cleansing, ${ }^{26}$ age, ${ }^{27,28}$ diet, or exercise. ${ }^{28}$ In patients with active IBD, the presence of mucus or blood can vary widely between bowel movements, and could therefore influence FC levels. ${ }^{26,28}$ Stool consistency and the time of the day of collection have been reported to influence variability, with opposite results according to some studies. Due to this intra-individual variability, FC-based decision-making strategies based on single measurements of FC might be wrong. Hence, there is a need to better grasp the intra-individual variability to ensure correct interpretation of a single measurement during IBD follow-up.In most laboratories, FC measurement is currently done on a single stool punch defined as a small random sample of approximately $100 \mathrm{mg}$ of faeces taken anywhere in the stool sample. An even distribution of calprotectin in the faeces is crucial to ensure the reliability of the test. However, there are no data about the reliability of a single stool punch of $100 \mathrm{mg}$. There is a growing interest in stool extraction methods and intrastool variability, because they might be relevant to FC-based decision-making strategies.

The increasing use of FC in clinical practice involves not only the clinical evaluation of disease activity on the dichotomous positive or negative interpretation of FC based on a single manufacturer's cut-off value, or a calculated cut-off value. Indeed, a modification of FC over time within the same patient is currently used in clinical practice as a marker of response to treatment or relapse.

\section{Methods}

\subsection{Aim of the study}

The aim of this study was to assess FC variability by evaluating intra- and intervariability of FC in a cohort of IBD and IBS patients [namely controls]. Intravariability included intrastool and intra-individual variability. The primary objective was to determine whether there is significant FC variability: [1] within a single bowel movement [intrastool variability]; [2] between two different bowel movements within a 1-6 days period [intra-individual variability]; and [3] between IBD patients [interindividual variability]. The secondary objective was to assess the robustness of using a single stool punch for FC measurement. Therefore, evaluation of intrastool variability was useful to determine whether FC is evenly distributed within the stool sample.

\subsection{Study design and ethical considerations}

This observational case-control study with prospective stool collection and retrospective analyses of the patient records was conducted in a single IBD tertiary referral centre, Erasme University Hospital [Université Libre de Bruxelles, Brussels, Belgium], after Ethics Committee agreement [reference number: 2014/229, approval date: June 17, 2014]. Each patient gave informed consent in accord with ethical guidelines of the 1975 Declaration of Helsinki. Patients with an established diagnosis of IBD or IBS were invited to participate in the study between March 2014 and August 2016.

\subsection{Definitions}

Intrastool variability was defined as FC variability between three punches in the same stool sample. Intra-individual variability was defined as FC variability between two stool samples collected a few days apart from the same patient. Interindividual variability was defined as the variability between IBD patients who differ by disease type [CD/UC], location [ileal/ileocolonic, and colonic $\mathrm{CD}$ ] or activity [quiescent/active]. A punch was defined as a small random sample of approximately $100 \mathrm{mg}$ of faeces taken anywhere in the stool sample, and a homogenate as a sample of 5 to $10 \mathrm{~g}$ of stool.

\subsection{Data collection}

All stool samples were collected prospectively. Clinical, endoscopic, and histological data were collected retrospectively by electronic chart review. Disease activity at the time of collection of the stool samples in IBD patients was assessed by clinical activity scores (Harvey-Bradshaw Index [HBI] in $\mathrm{CD}^{29}$ and partial Mayo score in UC), C-reactive proyein [CRP] levels, and endoscopic activity scores [presence/absence of ulcers for CD, endoscopic Mayo subscore in UC] when available. Active disease was defined as a Mayo score $>2$ for UC [3-5: mild; 6-10: moderate; 11-12: severe] or in the presence of endoscopic ulcers for CD. If a reliable endoscopy was not available, active disease was defined as a partial Mayo score $>1$ for UC [2-4: mild; 5-6: moderate; 7-9: severe] or a HBI >4 [5-7: mild; 8-16: moderate; >16: severe], or CRP above the normal range for $\mathrm{CD}$. An endoscopy is defined as reliable for disease activity evaluation in case of quiescent disease if performed up to 2 years before stool sampling without treatment modification [escalation or de-escalation] or clinical relapse [based on symptoms and/or CRP levels]. In case of active disease, criteria are more stringent in terms of timing and it is then a case-by-case evaluation according to the changes in treatment occurring around the stool sampling. 


\subsection{Stool samples}

Patients were instructed to collect two stool samples from two bowel movements within a 1 to 6 days interval, and to either return the samples to the hospital on the day of collection, or to store them at $4^{\circ} \mathrm{C}$ temperature and return them within maximum 5 days of collection. The presence of visible blood was recorded. Stool sampling was not allowed to be done during bowel cleansing or within $48 \mathrm{~h}$ of a colonoscopy, to avoid the impact of bowel cleansing on FC levels. ${ }^{26}$ All samples were stored at $-20^{\circ} \mathrm{C}$ before $\mathrm{FC}$ extraction, and measurements were done within 3 months of storage.

\subsection{Faecal calprotectin extraction and measurement}

Extraction was performed with both the conventional method [Roche device] and the homogenate method [see Supplementary materials, available as Supplementary data at ECCO-JCC online, for more precision about these methods]. FC was extracted from three punches of the first stool sample [Day 0] of more or less $100 \mathrm{mg}$ of stool, and on two homogenates [Day 0 and Day 1-6] of $5 \mathrm{~g}$ to $10 \mathrm{~g}$ of stool, depending on the quantity of faecal material available. For both sample types, i.e. punch and homogenate, the volume of extraction buffer was adapted to the stool weight so that the ratio weight/ volume remained constant for all stool samples. Extracts were analysed using EliA Calprotectin fluorescence enzyme immunoassay on the Phadia ${ }^{\circledR} 250$ [Thermo Fisher Scientific, Groot-Bijgaarden, Belgium] following manufacturer's instructions. Proposed cut-off value is $\leq 50 \mu \mathrm{g} / \mathrm{g}$. Measuring ranges were $15-3000 \mu \mathrm{g} / \mathrm{g}$. Samples scoring $<15 \mu \mathrm{g} / \mathrm{g}$ were registered as $5 \mu \mathrm{g} / \mathrm{g}$ and those scoring $\geq 3000 \mu \mathrm{g} / \mathrm{g}$ as $3000 \mu \mathrm{g} / \mathrm{g}$.

\subsection{Statistical analyses}

Data were analysed using MedCalc Statistical Software [version 18.5, MedCalc Software bvba, Ostend, Belgium]. Continuous variables were reported as medians with interquartile ranges [IQR] or ranges [minimum-maximum]. Categorical variables were reported as numbers $[n]$ and proportions [\%]. Comparisons of continuous variables were performed using a Mann-Whitney or Kruskal-Wallis test. Comparisons of categorical variables were performed using a Fisher's exact test or Pearson chi square test for trend. Extraction methods comparison for laboratory use of the homogenate was performed using the non-parametric Spearman rank correlation coefficient, Bland-Altman plot and the Passing-Bablok regression analysis. To compare FC values of different punches or homogenates from the same stool sample or from different stool samples, a Wilcoxon test was used. Intraclass correlation coefficients [ICC], which represent the degree of consistency among measurements, were calculated with $95 \%$ confidence interval $[\mathrm{CI}]$. This would be equal to 1 if there was no within-stool variation. To measure variability, coefficients of variation $[\mathrm{CV}]$ were calculated. To assess intrastool variability, coefficients of variation $[\mathrm{CV}]$ of the $\mathrm{FC}$ values of the three stool punches and the homogenate from the same sample from each patient were calculated. To assess intra-individual variability, CVs of the two samples within a 1 to 6 days interval from each patient were calculated. A $p$-value of $<0.05$ was considered statistically significant.

\section{Results}

\subsection{Study population}

A total of 122 patients were enrolled between March 2014 and August 2016. Two patients were excluded due to failure to return stool samples; 120 patients were included in the study for a total of 260 stool samples collected; 18 patients were included twice at 6 months to 1 -year interval for a total of 138 inclusions; $16 \mathrm{pa}$ tients failed to return the second stool sample a few days apart. Due to sometimes insufficient faecal material, all analyses (three punches and one homogenate on the first stool sample [Day 0] and one homogenate on the second stool sample [Day 1-6]) were not always possible.

The demographics of the study population and baseline characteristics of the IBD patients are summarised in Tables 1 and 2 . Among the $77 \mathrm{CD}$ patients, 24 had ileal disease, 40 ileocolonic disease, and 13 colonic disease. In UC patients, 20 had left UC and 14 had pancolic UC. Endoscopic assessment was available in 111/130 [85\%] IBD inclusions.

\subsection{Intravariability}

\subsubsection{Intrastool variability}

To determine whether FC was evenly distributed within the stool and to assess the representativeness of small random stool samples [punches], FC levels in three punches from the same first stool sample [Day 0], from a single bowel movement for each inclusion, were compared and calculation of the $\mathrm{CV}$ of the three punches was done. In all, 99 of the 138 first stool samples were considered for punches analyses; one sample was excluded due to insufficient faecal material; and 38 samples were excluded due to $\mathrm{FC}$ value below $15 \mu \mathrm{g} / \mathrm{g}$ or equal to or above $3000 \mu \mathrm{g} / \mathrm{g}$, that could have underestimated the variability. No statistical difference was shown in terms of FC value between the punches, either for the entire study population or within the six different subgroups of IBD patients [active ileal $\mathrm{CD}$, quiescent ileal $\mathrm{CD}$, active ileocolonic/colonic $\mathrm{CD}$, quiescent ileocolonic/colonic $\mathrm{CD}$, active $\mathrm{UC}$, and quiescent UC] [ $p>0.05]$. A strong correlation (ICC: $0.9148 ; 95 \%$ confidence interval [CI]: $0.8835-0.9392$ ) was found in terms of FC concentrations between the three punches. Median CVs were between $13 \%$ and $27 \%$, depending on the subgroup of patients with a median CV of $17 \%$ [range: 3-113] for the entire study population [Supplementary Table 1, available as Supplementary data at ECCO-JCC online]; $39 \%$ of the patients had a CV below $10 \%$ and $61 \%$ were above $10 \%$. There was no statistical difference in the

Table 1. Demographics of the study population.

\begin{tabular}{|c|c|c|c|c|c|}
\hline & $\mathrm{CD}[n=77]$ & $\mathrm{UC}[n=34]$ & IBS $[n=9]$ & All patients $[n=120]$ & $p$-value* \\
\hline Age [yr], median [range] & $36[15-83]$ & 37 [23-74] & 28 [16-62] & $36[15-83]$ & 0.64 \\
\hline Male, $n[\%]$ & $27[35]$ & $14[41]$ & $3[33]$ & $44[37]$ & 0.81 \\
\hline Disease duration [yr], median [IQR] & $7[4-14]$ & $3[1-7]]$ & $\mathrm{NA}$ & $6[2-11] n=111$ & $<0.01$ \\
\hline Current smoker, $n[\%]$ & $26[34]$ & $5[15]$ & $2[22]$ & $33[27]$ & 0.11 \\
\hline
\end{tabular}

CD, Crohn's disease; UC, ulcerative colitis; IBS, irritable bowel syndrome; $n$, number of patients; yr, years; IQR, interquartile range; NA, not applicable.

" $p$-value comparing different outcomes. Bold values are significant. 


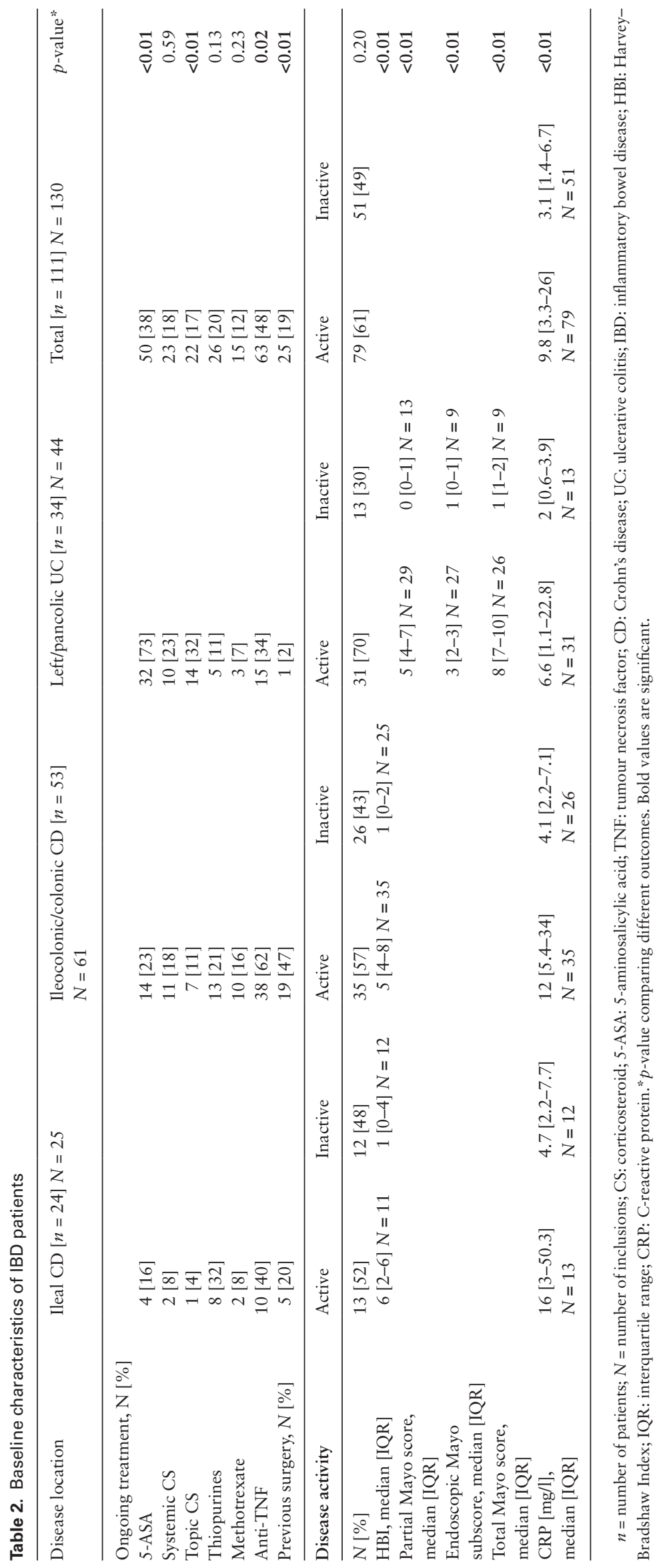


CVs, either between the seven subgroups of patients $[p=0.5744]$ or between CD and UC [ $p=0.7079]$, or between active and quiescent disease [ $p=0.3352$ ], or between low FC values [when at least two of the three punches were $<250 \mu \mathrm{g} / \mathrm{g}$ ] compared with higher FC values $[\geq 250 \mu \mathrm{g} / \mathrm{g}][p=0.8758]$. FC standard deviations correlated with mean FC levels [Spearman's coefficient of rank correlation $=0.854, p<0.0001]$. The variation was most pronounced in patients with high levels of FC [Figure 1]. Disease type, location, activity, and FC levels do not influence intrastool variability.

\subsubsection{Intra-individual variability}

To evaluate intra-individual variability, FC values of two stool samples from two different bowel movements, a few days apart for each inclusion, were compared and CVs between the two homogenates were calculated; 80 of the 122 inclusions that provided two stool samples were considered for analyses; 10 were excluded due to insufficient faecal material; and 32 were excluded due to FC value below $15 \mu \mathrm{g} / \mathrm{g}$ or equal to or above $3000 \mu \mathrm{g} / \mathrm{g}$, that could have underestimated the variability. No statistical difference was shown in terms of FC value between the homogenates a few days apart, either for the entire study population or within the six different subgroups of IBD patients $[p>0.05]$. ICC for single measures between the two homogenates was 0.7868 [95\% CI: $0.6863-0.8578]$ and Spearman rank correlation coefficient was 0.743 [ $p<0.0001$ ] [Figure 2]. Median CVs of the two samples were between $27 \%$ and $58 \%$, depending on the subgroup of patients with a median CV of $36 \%$ [range:0-123] for the entire study population [Supplementary Table 2, available as Supplementary data at ECCO-JCC online]; $14 \%$ of the patients had a CV below $10 \%$ and $86 \%$ were above $10 \%$. We did not show any statistical difference in the CVs between the six subgroups of patients [ $p=0.8337]$. FC standard deviations correlated with mean

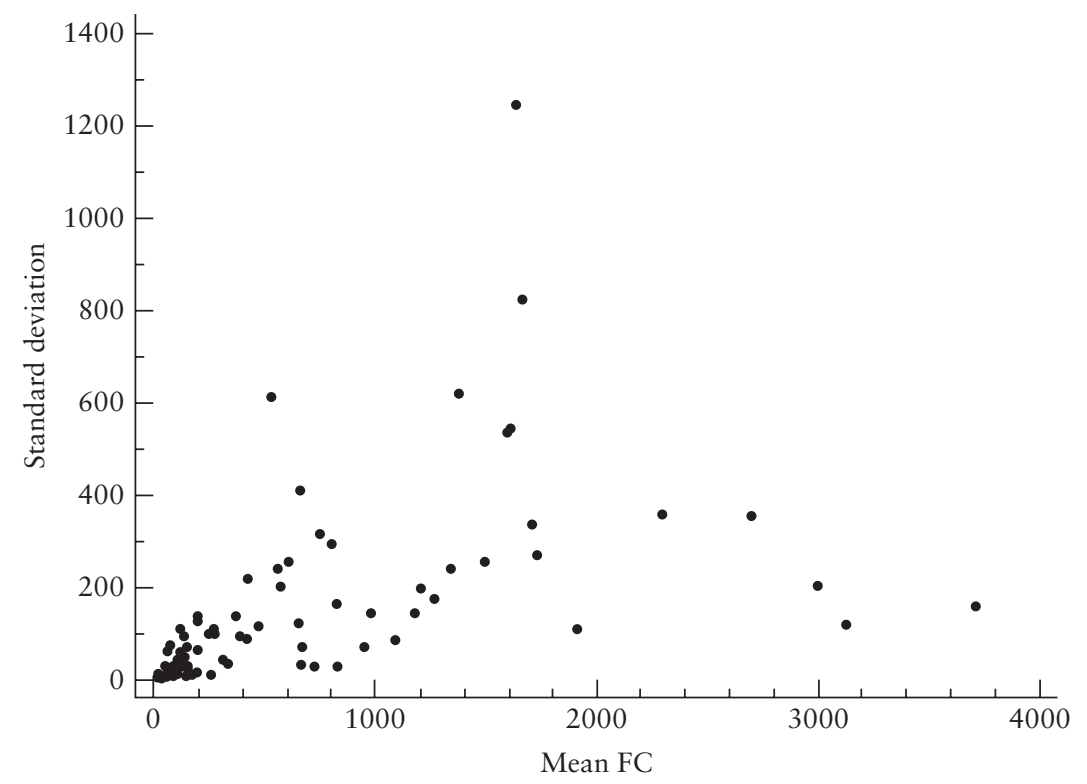

Figure 1. Correlation between mean faecal calprotectin $[F C]$ concentrations of the three punches and the corresponding standard deviations.

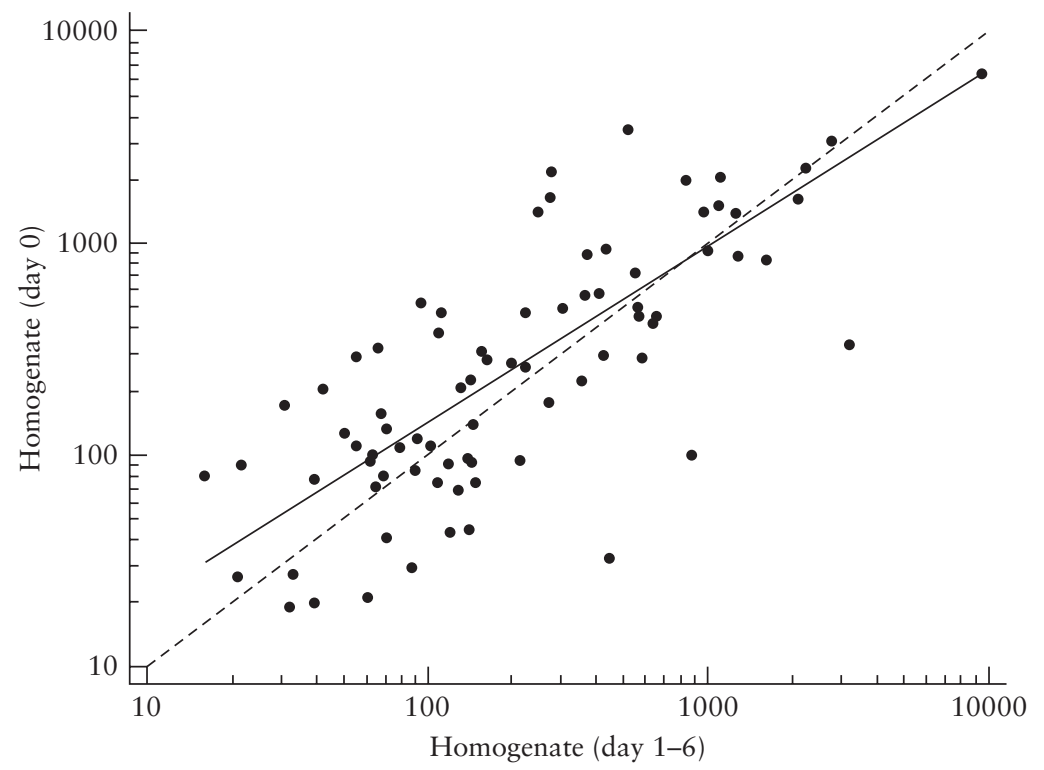

Figure 2. Comparison plot of faecal calprotectin [FC] concentrations in $\mu \mathrm{g} / \mathrm{g}$ of the two homogenates a few days apart. The continuous line is the best fitted linear line, and the dotted line is $\mathrm{y}=\mathrm{x}$ and represents the ideal result where sample $1=$ sample 2 . Spearman rank correlation coefficient was 0.743 [ $p<0.0001$ ]. 
FC levels [Spearman's coefficient of rank correlation $=0.781$, $p<0.0001]$. CVs of the two homogenates a few days apart were significantly higher compared with CVs of the three punches from the first stool sample $[p=0.0001]$.

\subsection{Intervariability}

Intervariability was evaluated between IBD patients who differed by disease type $[\mathrm{CD} / \mathrm{UC}$ ], location [ileal/colonic], or activity [quiescent/ active] and IBS patients [defined as controls]. Median FCs for each group are represented in Table 3 and Figure 3. All IBS patients had FC below $29 \mu \mathrm{g} / \mathrm{g}$. FC values were significantly higher in active UC compared with active $\mathrm{CD}[p=0.0031]$. The area under the curve for FC as a diagnostic test for IBD activity was 0.862 [ $p<0.0001]$, with a Youden index of 0.59 for a cut-off value of $122 \mu \mathrm{g} / \mathrm{g}$ [sensitivity $81 \%$, specificity $78 \%$ ]. CRP values were also significantly higher in active compared with quiescent IBD or IBS $[p<0.0001]$; see Supplementary data, available at ECCO-JCC online, for more details about comparison of FC and CRP to predict disease activity.

Table 3. Median FC.

\begin{tabular}{|c|c|c|c|c|}
\hline \multicolumn{2}{|c|}{ Groups } & \multirow{2}{*}{$\begin{array}{l}N \\
12\end{array}$} & \multirow{2}{*}{$\frac{\text { Median FC }[\mu \mathrm{g} / \mathrm{g}]}{17.5}$} & \multirow{2}{*}{$\begin{aligned} \text { Range } \\
5-643\end{aligned}$} \\
\hline 1 & Quiescent ileal CD & & & \\
\hline 2 & Active ileal CD & 13 & 490 & $59-1433$ \\
\hline 3 & Quiescent ileocolic/colic CD & 26 & 71 & $5-1247$ \\
\hline 4 & Active ileocolic/colic CD & 35 & 296 & $5-3000$ \\
\hline 5 & Quiescent UC & 13 & 21 & $5-656$ \\
\hline 6 & Active UC & 31 & 1614 & $5-3000$ \\
\hline 7 & IBS & 10 & 5 & $5-29$ \\
\hline \multicolumn{2}{|c|}{ Total } & 140 & 163 & $5-3000$ \\
\hline
\end{tabular}

CD: Crohn's disease; UC: ulcerative colitis; IBS: irritable bowel syndrome; FC: faecal calprotectin; $N=$ number of inclusions.

\section{Discussion}

The present observational case-control study revealed that a single stool punch of $100 \mathrm{mg}$ is reliable and representative of the entire stool sample for FC measurement, considering that intrastool variability is low and that a good correlation exists between the punches and the homogenate, a novel extraction method that assesses the robustness of using a single stool punch for FC measurement. However, CVs of two stool samples a few days apart [intra-individual variability] were significantly higher compared with CVs of the three punches, with median CVs ranging from $27 \%$ to $58 \%$ depending on the subgroup of IBD patients. Although intra-individual variability in FC levels occurs, the intrastool and intra-individual variation were not significant. The variability was not influenced by disease type, location, activity, or FC levels. It is of great importance to know the extent of FC variability to be able to correctly interpret the clinical significance of FC variation in the follow-up of IBD patients.

In most laboratories, FC measurement is currently done on a single stool punch. Even though volume-based extraction devices such as the faecal sample preparation kit [Roche device] [Roche Diagnostics $\mathrm{GmbH}[$, which bypass the step of weighting every single faecal sample, are nowadays widely used, the reference method is still the weighting method that was used in this study. An even distribution of calprotectin in the faeces is crucial to ensure the reliability of such test results. In the study of Roseth et al. ${ }^{21}$ the authors collected stool samples during $24 \mathrm{~h}$ and showed good correlation between three random stool samples of $5 \mathrm{~g}$ and the rest of the thoroughly blended stool, with a median CV of $21 \%$. However, a $5-\mathrm{g}$ sample is not comparable to a $100-\mathrm{mg}$ sample, and we were among the first to show the reliability of a single stool punch of $100 \mathrm{mg}$, even more in the different subgroups of patients for FC measurement with a median $\mathrm{CV}$ of $17 \%$. It is also important to stress that due to a lack of international standardisation for FC measurement and variability among FC measurement methods, it is recommended

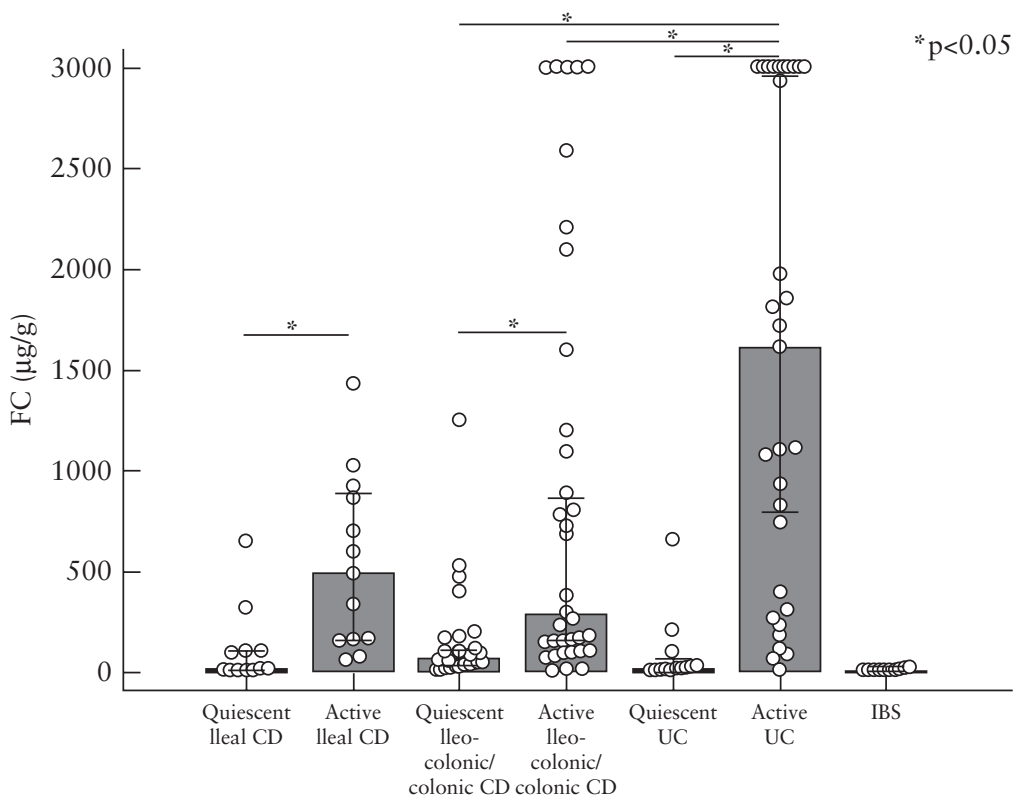

Figure 3. Faecal calprotectin [FC] values according to disease type, location, and activity. FC is expressed in medians and $95 \%$ confidence intervals for medians. 1 = quiescent ileal $C D ; 2=$ active ileal $C D ; 3=$ quiescent ileocolonic/colonic $C D ; 4=$ active ileocolonic/colonic $C D ; 5=$ quiescent $U C ; 6=$ active $U C ;$ and $7=I B S$ [controls]. [1] isdifferent from [2], [4], and [6]; [2] is different from [1], [3], [5], [6], and [7]; [3] is different from [2], [4], [6], and [7]; [4] is different from [1], [3], [5], [6], and [7]; [5] is different from [2], [4], and [6]; [6] is different from [1], [2], [3], [4], [5], and [7]; [7] is different from [2], [3], [4], and [6] [p<0.05]. CD, Crohn's disease; UC, ulcerative colitis. 
to use the same method to follow a specific patient because results from different methods are not commutable. ${ }^{30}$ Therefore, it is not recommended to extrapolate these results to other extraction devices or to other assays.

This study cohort is relatively large in comparison with other studies that have evaluated FC variability. Furthermore, active or inactive UC and CD patients, as well as a control group, were included in the present study, showing that the results are applicable for all IBD patients. Indeed in previous studies, significant variation in FC concentrations has been observed, mainly in subgroups of IBD patients [UC or CD with quiescent or active disease]. ${ }^{25,31-33}$

Our results in terms of intra-individual variability of FC are in agreement with other recent studies. ${ }^{25,31}$ Calafat et al. ${ }^{31}$ showed a median CV of $40 \%$ in samples collected during the same day, and Lasson et al. ${ }^{25}$ showed a median $\mathrm{CV}$ of $52 \%$ and a strong correlation in terms of FC concentrations between two random samples collected during the same day [within-day variation] with an ICC of 0.79 . Our median CV in samples collected a few days apart [day-to-day variation] was $36 \%$ with the same ICC of 0.79 .

Some points in our study could be criticised. No instructions were given to our patients as to the time of day when they were to collect their samples. Indeed, unlike other studies, we did not require a sample from the first bowel movement. Regarding this issue, Lasson et al. ${ }^{25}$ found that FC sampling should be taken from the first bowel movement of the morning, given a significant positive correlation between the level of FC and the time between bowel movements. However, Kristensen et al. ${ }^{33}$ did not show significant intra-individual diurnal variation, even though this was on a limited number of patients $[n=50]$. In the same way, Calafat et al. ${ }^{31}$ showed that the timing of stool sampling does not seem to be relevant, because the highest or lowest FC values may also vary during the day. Du et al., ${ }^{32}$ although suggesting that the morning sample provided the lowest clinically significant intrastool FC variability, did not show any difference in terms of intrastool variability depending on the time of the day. Given the results of those studies, there is actually no demonstrated benefit in sampling the first stool in the morning for FC measurement. Moreover, we did not assess some macroscopic characteristics of stools, such as mucus or consistency, that might influence FC levels. However, Lasson et al. ${ }^{25}$ were not able to find a correlation between stool consistency, content of blood, or time between bowel movements and CV. However, sampling error due to, for example, stool with non-homogeneous distribution of mucus or blood, was controlled by the fact that FC concentrations of the homogenates were very well correlated with the punches, which challenges the assumption that non-homogeneous distribution of mucus or blood may have an impact on FC variability.

In conclusion, the present study reveals that FC is a good marker of activity in IBD, in CD and in UC, regardless of location [ileal and ileocolonic/colonic CD]. A single stool punch is reliable for FC measurement, considering that intrastool variability is low. Intra-individual variability a few days apart is significantly higher. Therefore, decision-making strategies based on single measurements should take into account this variability, so as to determine the minimum optimal variation to be achieved in treat-to target strategy, rather than a cut-off, especially in high FC levels.

\section{Funding}

This work was supported by the Fonds Erasme for Medical Research [Doctoral research fellow grant to $\mathrm{AC}$. This work was alsosupported by the Belgian
National Fund of Scientific Research [FNRS] [Doctoral Research Fellow grant to $\mathrm{CL}$; and DF is Research Director of FNRS].

\section{Conflict of Interest}

All authors have no conflict of interest to disclose. The authors did not have any writing assistance in producing this manuscript.

\section{Acknowledgments}

All reagents were provided by Thermofisher [Thermo Fisher Diagnostics, BE-1702 Groot-Bijgaarden, Belgium]. Thermofisher did not have any role in the collection, analysis, and interpretation of data, in the writing of the manuscript, or in the decision to submit the manuscript for publication.

\section{Author Contributions}

All authors contributed to the concept and design of the study; PS and JS coordinated processing of samples; JK, MRB, and FB analysed the stool samples; collection, analysis, and interpretation of the data were performed by AC and JK; AC and JK performed the statistical analysis; AC made the draft of the manuscript with contribution from DF; all authors carried out critical revision of the manuscript for important intellectual content; all authors read and approved the final version of the manuscript.

\section{Supplementary Data}

Supplementary data are available at ECCO-JCC online.

\section{References}

1. Røseth AG, Aadland E, Jahnsen J, Raknerud N. Assessment of disease activity in ulcerative colitis by faecal calprotectin, a novel granulocyte marker protein. Digestion 1997;58:176-80.

2. Abraham BP, Kane S. Fecal markers: calprotectin and lactoferrin. Gastroenterol Clin North Am 2012;41:483-95.

3. Lin J-F, Chen J-M, Zuo J-H, et al. Meta-analysis. Inflamm Bowel Dis 2014;20:1407-15.

4. Konikoff MR, Denson LA. Role of fecal calprotectin as a biomarker of intestinal inflammation in inflammatory bowel disease. Inflamm Bowel Dis 2006;12:524-34.

5. Røseth AG, Schmidt PN, Fagerhol MK. Correlation between faecal excretion of indium-111-labelled granulocytes and calprotectin, a granulocyte marker protein, in patients with inflammatory bowel disease. Scand J Gastroenterol 1999;34:50-4.

6. Berni Canani R, Rapacciuolo L, Romano MT, et al. Diagnostic value of faecal calprotectin in paediatric gastroenterology clinical practice. Dig Liver Dis 2004;36:467-70.

7. Lobatón T, Rodríguez-Moranta F, Lopez A, Sánchez E, RodríguezAlonso L, Guardiola J. A new rapid quantitative test for fecal calprotectin predicts endoscopic activity in ulcerative colitis. Inflamm Bowel Dis 2013;19:1034-42.

8. Jensen MD, Kjeldsen J, Nathan T. Fecal calprotectin is equally sensitive in Crohn's disease affecting the small bowel and colon. Scand J Gastroenterol 2011;46:694-700.

9. Reenaers C, Bossuyt P, Hindryckx P, Vanpoucke H, Cremer A, Baert F. Expert opinion for use of faecal calprotectin in diagnosis and monitoring of inflammatory bowel disease in daily clinical practice. United European Gastroenterol J 2018;6:1117-25.

10. Guardiola J, Lobatón T, Rodríguez-Alonso L, et al. Fecal level of calprotectin identifies histologic inflammation in patients with ulcerative colitis in clinical and endoscopic remission. Clin Gastroenterol Hepatol 2014;12:1865-70.

11. Mao R, Xiao YL, Gao X, et al. Fecal calprotectin in predicting relapse of inflammatory bowel diseases: a meta-analysis of prospective studies. Inflamm Bowel Dis 2012;18:1894-9. 
12. Tibble JA, Sigthorsson G, Bridger S, Fagerhol MK, Bjarnason I. Surrogate markers of intestinal inflammation are predictive of relapse in patients with inflammatory bowel disease. Gastroenterology 2000;119:15-22.

13. De Vos M, Louis EJ, Jahnsen J, et al. Consecutive fecal calprotectin measurements to predict relapse in patients with ulcerative colitis receiving infliximab maintenance therapy. Inflamm Bowel Dis 2013;19:2111-7.

14. Sipponen T, Savilahti E, Kolho KL, Nuutinen H, Turunen U, Färkkilä M. Crohn's disease activity assessed by fecal calprotectin and lactoferrin: correlation with Crohn's disease activity index and endoscopic findings. Inflamm Bowel Dis 2008;14:40-6.

15. Molander P, af Björkesten CG, Mustonen H, et al. Fecal calprotectin concentration predicts outcome in inflammatory bowel disease after induction therapy with TNF $\alpha$ blocking agents. Inflamm Bowel Dis 2012;18:2011-7.

16. De Vos M, Dewit O, D'Haens G, et al.; on behalf of BIRD. Fast and sharp decrease in calprotectin predicts remission by infliximab in anti-TNF naïve patients with ulcerative colitis. J Crohns Colitis 2012;6:557-62.

17. Costa F, Mumolo MG, Ceccarelli L, et al. Calprotectin is a stronger predictive marker of relapse in ulcerative colitis than in Crohn's disease. Gut 2005;54:364-8.

18. Schoepfer AM, Beglinger C, Straumann A, Trummler M, Renzulli P, Seibold F. Ulcerative colitis: correlation of the Rachmilewitz endoscopic activity index with fecal calprotectin, clinical activity, C-reactive protein, and blood leukocytes. Inflamm Bowel Dis 2009;15:1851-8.

19. Wagner M, Peterson CG, Ridefelt P, Sangfelt P, Carlson M. Fecal markers of inflammation used as surrogate markers for treatment outcome in relapsing inflammatory bowel disease. World J Gastroenterol 2008;14:5584-9; discussion 5588.

20. Sipponen T, Björkesten CG, Färkkilä M, Nuutinen H, Savilahti E, Kolho KL. Faecal calprotectin and lactoferrin are reliable surrogate markers of endoscopic response during Crohn's disease treatment. Scand J Gastroenterol 2010;45:325-31.

21. Røseth AG, Fagerhol MK, Aadland E, Schjønsby H. Assessment of the neutrophil dominating protein calprotectin in feces. A methodologic study. Scand J Gastroenterol 1992;27:793-8.

22. Kopylov U, Rosenfeld G, Bressler B, Seidman E. Clinical utility of fecal biomarkers for the diagnosis and management of inflammatory bowel disease. Inflamm Bowel Dis 2014;20:742-56.
23. Moum B, Jahnsen J, Bernklev T. Fecal calprotectin variability in Crohn's disease. Inflamm Bowel Dis 2010;16:1091-2.

24. Naismith GD, Smith LA, Barry SJ, et al. A prospective single-centre evaluation of the intra-individual variability of faecal calprotectin in quiescent Crohn's disease. Aliment Pharmacol Ther 2013;37:613-21.

25. Lasson A, Stotzer PO, Öhman L, Isaksson S, Sapnara M, Strid H. The intra-individual variability of faecal calprotectin: a prospective study in patients with active ulcerative colitis. J Crohns Colitis 2015;9:26-32.

26. Kolho KL, Alfthan H, Hämäläinen E. Effect of bowel cleansing for colonoscopy on fecal calprotectin levels in pediatric patients. J Pediatr Gastroenterol Nutr 2012;55:751-3.

27. Joshi S, Lewis SJ, Creanor S, Ayling RM. Age-related faecal calprotectin, lactoferrin and tumour M2-PK concentrations in healthy volunteers. Ann Clin Biochem 2010;47:259-63.

28. Poullis A, Foster R, Shetty A, Fagerhol MK, Mendall MA. Bowel inflammation as measured by fecal calprotectin: a link between lifestyle factors and colorectal cancer risk. Cancer Epidemiol Biomarkers Prev 2004;13:279-84.

29. Vermeire S, Schreiber S, Sandborn WJ, Dubois C, Rutgeerts P. Correlation between the Crohn's Disease Activity and Harvey-Bradshaw indices in assessing Crohn's disease severity. Clin Gastroenterol Hepatol 2010;8:357-63.

30. Labaere D, Smismans A, Van Olmen A, et al. Comparison of six different calprotectin assays for the assessment of inflammatory bowel disease. United European Gastroenterol J 2014;2:30-7.

31. Calafat M, Cabré E, Mañosa M, Lobatón T, Marín L, Domènech E. High within-day variability of fecal calprotectin levels in patients with active ulcerative colitis: what is the best timing for stool sampling? Inflamm Bowel Dis 2015;21:1072-6.

32. Du L, Foshaug R, Huang VW, et al. Within-stool and within-day sample variability of fecal calprotectin in patients with inflammatory bowel disease: a prospective observational study. J Clin Gastroenterol 2018;52:235-40.

33. Kristensen V, Malmstrøm GH, Skar V, Røseth A, Moum B. Clinical importance of faecal calprotectin variability in inflammatory bowel disease: Intra-individual variability and standardisation of sampling procedure. Scand J Gastroenterol 2016;51:548-55. 